\title{
Highly sensitive surface plasmon resonance optical detection of ferric ion using CTAB/hydroxylated graphene quantum dots thin film
}

\begin{abstract}
The development of optical sensors for heavy metal ion detection has been rapidly growing; unfortunately, the current methods suffer limitations that led to the emergence of an outstanding technique called surface plasmon resonance (SPR) spectroscopy. In this paper, the performance of the SPR optical sensor in detecting ferric ions (Fe3+) was successfully enhanced by depositing novel cetyltrimethylammonium bromide modified hydroxylated graphene quantum dots (CTAB/HGQDs) onto a gold $(\mathrm{Au})$ thin film using the spin coating technique. Upon exposure to $\mathrm{Fe} 3+$, the SPR responses of both CTAB/HGQDs thin film and bare Au thin film were compared and studied, emphasizing the sensitivity, binding affinity, full width at half maxima, signal-tonoise ratio, and data accuracy. The CTAB/HGQDs thin film achieved a high sensitivity value of $29.886^{\circ} \mathrm{ppm}-1$ for $\mathrm{Fe} 3+$ up to $0.1 \mathrm{ppm}$. The strong binding affinity was confirmed using the Langmuir isotherm model calculation. To the end, the CTAB/HGQDs thin film was characterized using atomic force microscopy for morphological study, confirming its interaction with Fe3+.
\end{abstract}

